\title{
Coflow - a step forward for solution SAXS
}

Nigel M. Kirby, Tim M. Ryan

\section{Australian Synchrotron, Clayton, VIC, Australia}

Small angle X-ray scattering (SAXS) is a useful tool for analysing protein structures that is becoming increasingly popular in structural biology, and is now commonplace at synchrotrons worldwide. SAXS has a number of advantages over other techniques, but radiation damage and sample consumption limit the utility of the technique to the wider protein community. On undulator beamlines, radiation damage is typically handled by flowing the sample past the beam, but its effectiveness is poor due to the well-known laminar flow profile inside sample cells. Low flow velocities near the edges of sample cells result in highly elevated localised radiation dose and damage, which reduces the efficiency of the measurement overall.

Through modelling and experimentation we have devise an improved method for solution SAXS which avoids this problem by limiting the sample flow to the centre of the sample cell surrounded by a flowing sheath fluid [1]. The X-ray beam and flow velocity distributions provide a much uniform dose distribution than conventional flowing analysis, allowing sensitive protein solutions to be exposed to at least 10 fold greater flux despite lower sample flow rates. The high efficiency of X-ray delivery means SAXS measurements can now be routinely performed much closer to the fundamental limits imposed by the sample's radiation resistance. The geometry of the method is well suited to high intensity, small x-ray beams on low-emittance x-ray sources: for example the Australian SAXS/WAXS beamline [2] used to be restricted to only a small fraction of its full flux for solution bioSAXS experiments, but is now typically flux-limited in routine operations. The large increase in usable incident flux increases the obtainable data quality per unit volume of sample, and/or helps Users analyse more dilute or smaller samples. The kinetics of the measurement can eliminate aggregation as a source of observed radiation damage. Using a direct injection geometry, the method can collect data on microliter sample volumes. Using a matched buffer for the sheath flow maintains accurate background subtraction for very dilute and small volume samples. Segregation between the inner protein solution and the capillary wall by the sheath flow prevents fouling of capillaries by unstable or radiation damaged proteins.

Whilst the protein flow stream experiences fast, relatively uniform flow and tightly controllable Xray dose, the outer buffer stream which sees the slow flowing boundary condition must withstand high doses near the capillary walls. For coflow under high flux conditions it has been essential to comprehensively investigate radiation damage of buffers, to obtain strategies for producing buffers able to withstand extreme doses during extended exposure. This work may benefit conventional solution bioSAXS work where effects of radiation damage of buffers is more subtle and may be harder to spot.

Coflow is well suited to routine, high throughput bioSAXS, particularly for size exclusion chromatography on fast-running columns which might produce elution peaks only a few seconds wide, that can really take advantage of the high $\mathrm{X}$-ray flux the method allows. The method is now standard on the Australian SAXS/WAXS beamline. Coflow's benefits in data quality, sample volume and lack of fouling by unstable samples helps SAXS continue to grow in its utility and impact in challenging new areas of biology. This presentation will show the core developments behind the method and some illustrative examples of its use.

\section{References}

[1] N. Kirby, N. Cowieson, A.M. Hawley, S.T. Mudie, D.J . McGillivray, M. Kusel, V.Samardzic-Bobana and T.M. Ryan, "Improved radiation dose efficiency in solution SAXS using a sheath flow sample environment", Acta Cryst. (2016) D72, 1254-1277. doi: 10.1107/S2059798316017174

[2] N.M. Kirby, S.T. Mudie, A.M. Hawley, D.J. Cookson, H.D.T. Mertens, N. Cowieson and V. Samardzic-Boban, "A low-background-intensity focusing small-angle X-ray scattering undulator beamline", J . Appl. Cryst. (2013). 46, 16701680. doi: 10.1107/S002188981302774X 